\title{
Comparison of Observed VLF Attenuation Rates and Excitation Factors With Theory
}

\author{
A. D. Watt and R. D. Croghan
}

\author{
Contribution From DECO Electronics, Inc., Boulder Division, Boulder, Colo.
}

\begin{abstract}
The properties of VLF propagating modes are briefly reviewed and simplified equations are presented which can be employed in calculating the fields produced. Experimentally determined excitation factors are compared with theoretical curves obtained by Wait and found to agree rather closely. Attenuation rates are shown as a function of frequency and are found to agree rather closely with calculated values using a proposed simplified perturbation solution for attenuation rates based on reflection coefficients at the ground and ionosphere. When employing values of ionospheric reflection coefficients recently presented by Wait and Walters, the nonreciprocal effects in attenuation rate are found both theoretically and experimentally to be much greater in the $10 \mathrm{kc} / \mathrm{s}$ region than in the 20 to $30 \mathrm{kc} / \mathrm{s}$ region. Finally, experimental field strength versus distance data are compared with mode calculations and found to compare well all the way from 1 megameter out to and including fields at the antipode (20 megameters).
\end{abstract}

\section{Introduction}

The majority of VLF transmitting installations employ frequencies lying within the 10 to $30 \mathrm{kc} / \mathrm{s}$ region. Within this frequency region, and particularly at great distances, most of the energy can be considered as being propagated as a guided wave in the space between the earth and a conducting ionosphere. Observed electromagnetic fields within the earth-ionosphere cavity indicate that the actual physical case can be well approximated with a model such as shown in figure 1 where the earth is considered as highly conducting with a reflection coefficient of +1 and the ionosphere is highly reflective with a reflection coefficient of approximately -1 . For these idealized assumptions, the first order transverse magnetic $\left(\mathrm{TM}_{1}\right)$ wave will have electrical field lines similar to those shown on the left hand side of the figure. The manner in which the magnitude of the vertical electric field, $E_{z}$, and the horizontal electric field, $E_{y}$, vary with height, $z$, is shown on the lower left hand side of the figure. The scale chosen for this figure is representative of what is expected with a frequency of $15 \mathrm{kc} / \mathrm{s}$. On the right hand side of the figure are shown the electric field lines for the $\mathrm{TM}_{2}$; i.e., the second order transverse magnetic wave. The amplitude-versus-height variations of the vertical electric and horizontal electric fields are again indicated at the bottom of the figure. The electric field lines are only shown within the cavity and no attempt is made to indicate the field lines lying outside the boundaries in the actual physical case where conductivities are not infinite.

For distances where near field effects can be neglected, Wait [1962, p. 199] has shown that the vertical electric field $E_{z}$ can be represented in integral form or as the summation of an infinite number of modes. In the practical case for distances less than about $1 \mathrm{Mm}$, the total electric field at VLF can be well approximated as the sum of the groundwave and one or two sky reflected rays. This field is

$$
E_{z}=E_{z, g}+\sum_{m=1}^{m=2 \text { or } 3} E_{z, m}
$$

$\lambda / 2<d<1 \mathrm{Mm}$

where $E_{z, g}$ is the groundwave contribution and $E_{z, m}$ is the $m$ th hop skywave contribution.

For distances greater than $1 \mathrm{Mm}$, the vertical electric field can now be represented as the sum of a relatively small number of waveguide modes ${ }^{1}$

$$
E_{z} \simeq \sum_{n=1}^{n=1} E_{z, n}
$$

$1 \mathrm{Mm}<d$.

The field produced for a given mode can be written in decible terms as

$$
\begin{aligned}
E_{z, n}[\mathrm{db}, \mathrm{v} / \mathrm{m}]= & 10 \log P_{r}+K_{n}^{\prime}-10 \log f-10 \log h_{\imath, t} \\
& +10 \log \Lambda_{n, t}-10 \log (a \sin d / a) \\
& -10 \log h_{i, r}+10 \log \Lambda_{n, r}+20 \log \\
& G_{h, n}-L_{d, n}-\alpha d / 10^{6}
\end{aligned}
$$

where: $E_{z, n}[\mathrm{db}, \mathrm{v} / \mathrm{m}]$ is the vertical electric field produced by the $n$th mode in db relative to $1 \mathrm{v} / \mathrm{m}$,

1 At VLF the $n=0$ mode which is actually a TEM mode is highly attenuated and is not expected to contribute to the observed fields. 


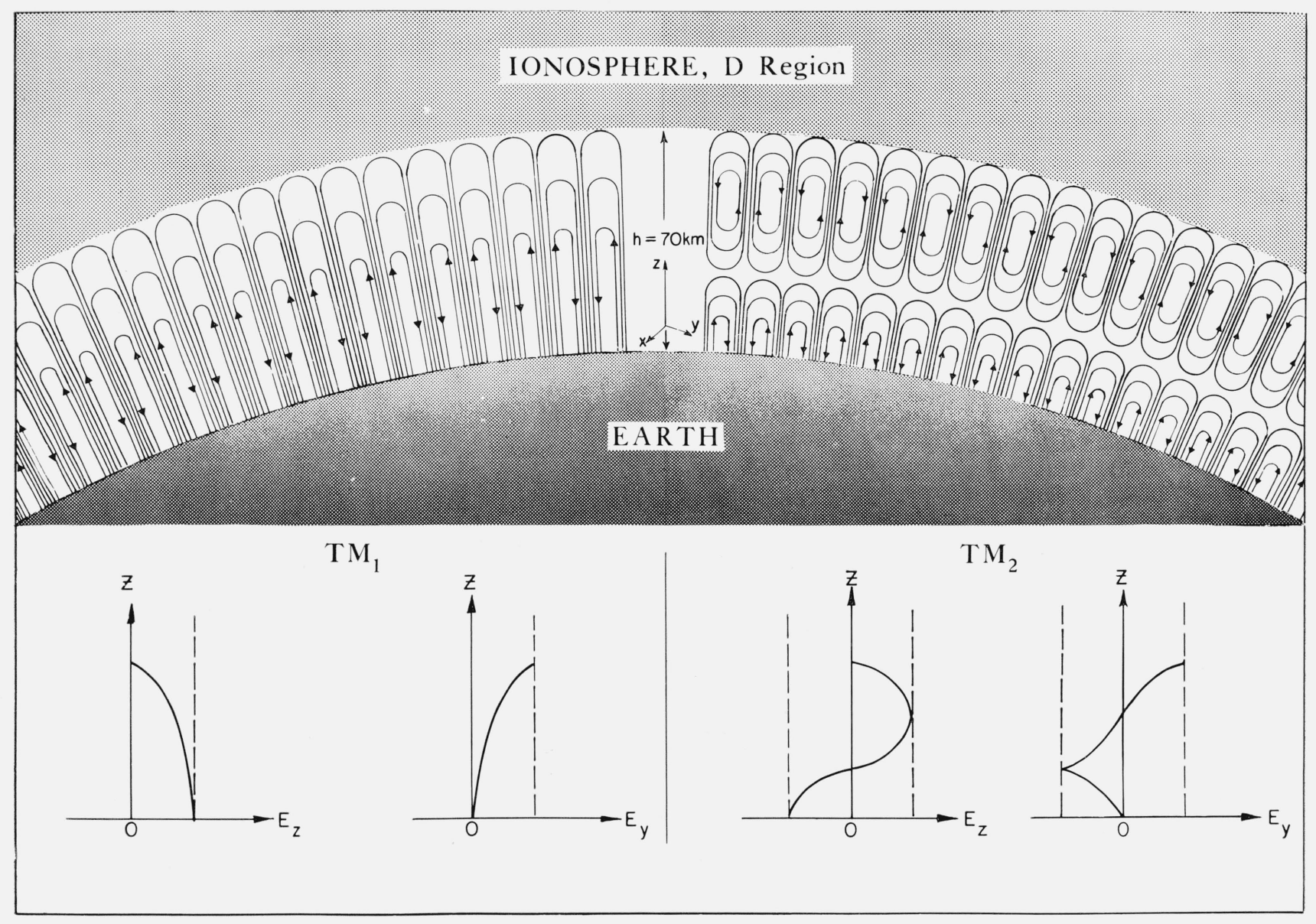

Figure 1. Electrical field structure for wave guide modes.

$P_{r}$ is the power radiated into the half space above the earth in watts, $K_{n}^{\prime}$ is a constant which relates field strengths at the surface to power radiated and is equal to 104.3 for modes of $n=1,2--, f$ is the frequency in cycles per second, $h_{i, t}$ and $h_{i, r}$ are the effective ionospheric heights at the transmitter and receiver locations, nominally $7 \times 10^{4} \mathrm{~m}$ for day and $9 \times 10^{4} \mathrm{~m}$ at night. $\Lambda_{n, t}$ is the mode excitation modification factor which is defined as the ratio of power launched into the concentric spherical shell guide relative to that for a flat guide with perfectly conducting earth, $a$ is the earths radius $\simeq 6.4 \times 10^{6} \mathrm{~m}, d$ is the path distance, $(a \sin d / a)$ accounts for energy spreading in the spherical guide structure, $\Lambda_{n, r}$ is the mode field modification factor for a curved earth at the ionospheric heights applicable at the receiver, $20 \mathrm{Log}$ $\mathrm{G}_{h, n}$ is the height gain factor in decibles relative to the field at the surface, i.e., $z=0, L_{d, n}$ is a factor in decibels which accounts for losses at discontinuities along the path, such as abrupt changes in surface conductivity, or in guide height such as can occur for a sunrise-sunset boundary, and $\alpha$ is the effective attenuation rate in $\mathrm{db} / \mathrm{Mm}$ which is normally different for each mode number.

As we will see later, this idealized model and associated equations is able to account rather closely for the observed variations in field strength for transmitters and receivers situated within the earth ionsophere cavity. ${ }^{2}$ The important problems faced in obtaining numerical values for the factors in (1.3) consist primarily in determining: (1) the efficiency of a given transmitting installation in launching radiofrequency energy into the half space above the earth, (2) the efficiency with which this energy couples into the propagating mode, (3) the manner in which the energy spreads throughout the concentric spherical shell cavity, (4) the height gain characteristic, (5) the discontinuity loss factor, and (6) the attenuation rate in the last term which accounts for losses along the path.

The effective radiated power $P_{r}$ can be determined from near field measurements applied to well-known relations. The frequency, of course, is known and the other geometrical factors involving distance, height to the ionosphere, and the spreading terms can be rather precisely determined. The principal re-

${ }_{2}$ Normally in the 1 to $10 \mathrm{Mm}$ distance region the fields are considered as being produced primarily by the $\mathrm{TM}_{2}$ and $\mathrm{TM}_{1}$ modes. The possibility of TE modes contributing must not be neglected particularly at short or very long distances 
maining unknowns relate to the excitation efficiency and attentuation rates.

In order to determine attenuation rates, Wait [1958] employed a technique in which he plotted the sum of the vertical electrical field in decibels relative to $1 \mathrm{v}$ per meter and the spreading term $10 \log (a \sin d / a)$, as a function of the distance. This technique has been employed by the authors for a large number of observed field strength versus distance contours to obtain both excitation factors and attenuation rates. A typical $E_{z}(a \sin d / a)$ versus $d$ curve is shown in figure 2 for the transmitting station of LCM of Stavanger, Norway operating at a frequency of $24.4 \mathrm{kc} / \mathrm{s}$. The data was taken by Round et al. [1925]. For a distance of $1 \mathrm{~km}$, the energy spreading term $(a \sin d / a)$ becomes equal to zero and if the radiated power, frequency, and ionospheric height are known, it is possible to arrive at a $1 \mathrm{~km}$ intercept point which for all practical purposes can be plotted for $d=0 \mathrm{Mm}$ (megameters) on the curve in figure 2. For this particular station, the calculated $d=0$ intercept value is 89.5 decibels while the actual intercept is seen to be some $9 \mathrm{db}$ below the flat earth calculation. Since the attenuation is negligible for the $1 \mathrm{~km}$ distance, the resulting field strength difference is actually equal to $20 \log \Lambda$ which is the total modification required for the spherical earth case; i.e., $20 \log \Lambda=10 \log \Lambda_{t}+10 \log \Lambda_{r}$ if the ionosphere height is the same at transmitter and receiver. The slope of the curve, since the distance spreading term has been added to the observed fields, is seen to be constant and for this particular case is equal to about $2.3 \mathrm{db} / \mathrm{Mm}$. The attenuation rate, $\alpha$, is determined by losses at the earth and ionosphere boundaries and will be shown later to be dependent upon many factors including: frequency, diurnal effects, seasonal effects, latitude effects, solar activ- ity, meteoric activity, direction of propagation relative to the earth's magnetic field, and earth's surface conductivity.

\section{Excitation Factors and Height Gain}

The power radiated into the half space above the ground surrounding the antenna is given by the well-known relation

$$
P_{r, \text { half space }}=I^{2} 160 \pi^{2}\left(h_{e} / \lambda\right)^{2}
$$

where $I$ is the rms antenna current, $h_{e}$ is the effective antenna height in meters, and $\lambda$ is the wavelength in meters. When the antenna is considered as being located between two parallel plates, the fields at a large distance from the source can be considered as though they were produced by a set of images producing an equivalent line current as shown by Wait [1960]. Employing the field produced by this equivalent line source, it can be shown that the power launched into a given spherical earth mode relative to the total half space radiated power is

$$
\frac{P_{r, n=(0,1,2 \ldots)}}{P_{r, \text { half space }}}=\frac{\eta_{0} \lambda \Lambda}{320 \pi h} \simeq \frac{1.12 \times 10^{8} \Lambda}{f h}
$$

where $\eta_{0}$ is the impedance in free space, $f$ is the frequency in cycles per second, $h$ is the ionospheric height in meters, and $\Lambda$ is the mode excitation modification factor. For a spherical earth, the variation in electromagnetic field strength with height changes from that of the flat earth case. This change in field strength with height modifies, by a factor $\Lambda$, the amount of energy which is coupled into the spherical cavity or concentric spherical cavity. In addition, the field strength observed at the surface by the receiver is reduced by a factor $\Lambda^{1 / 2}$ for a fixed

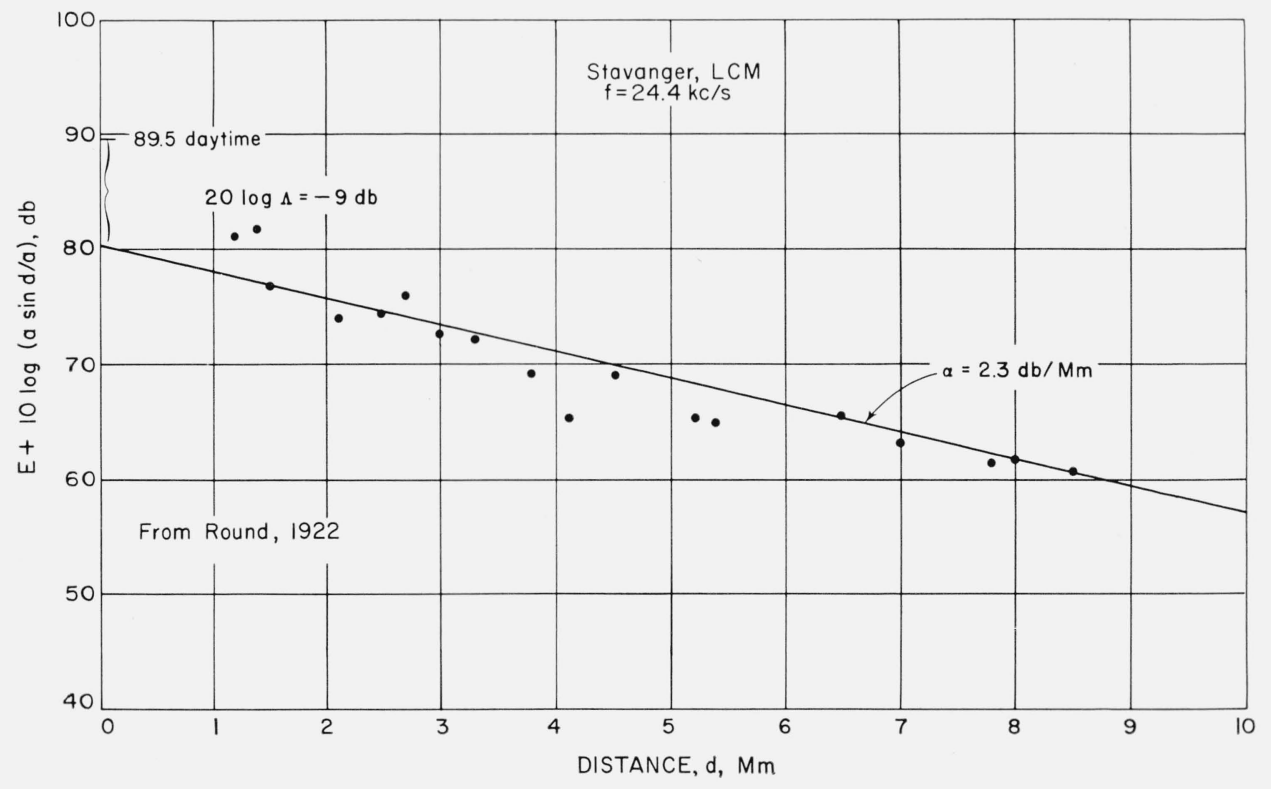

Figure 2. Typical E (a sin d/a) versus d curve. 
energy flow in the guide. The result, assuming $h_{i}$ is constant, is that the vertical electric field calculated for a flat earth must be modified by $\Lambda$ to the first power. Wait [1962] has derived values for the modification factor $\Lambda$ which are shown in figure 3 for a typical day time ionospheric height of $70 \mathrm{~km}$. The curves are for the first and second order modes where the earth and ionosphere boundaries are considered as perfectly reflecting.

In order to determine $\Lambda$ experimentally, a large number of field strength versus distance curves were plotted (in a manner similar to that shown in fig. 2) and the resulting experimental data points shown in figure 3 appear to group around the $n=1$ curve over quite a large frequency region. The few data points shown at the upper right hand side of the figure near the $n=2$ curve, were obtained from line intercepts which appeared to be representative of second order mode propagation. These line intercepts correspond to a rather high attenuation rate portion of the field strength versus distance curve that in general is only applicable out to several megameters from the source. As might be anticipated, these effects are seen essentially only at the higher frequencies; i.e., about $20 \mathrm{kc} / \mathrm{s}$ and up.

It is interesting to observe that on several occasions the excitation factors appear to lie appreciably below the theoretical curve. For example, at 21 $\mathrm{kc} / \mathrm{s}$ on one occasion the excitation factor was near the curve while for the other example shown, the excitation modification apparently produced was about $5 \mathrm{db}$ below the theoretical value. The particular field strength versus distance curve associated with this low excitation factor had a rather low attenuation rate, and it is likely that the propagation conditions were approaching the earth detached case as described by Wait [1962] where most of the energy is concentrated near the ionosphere and any

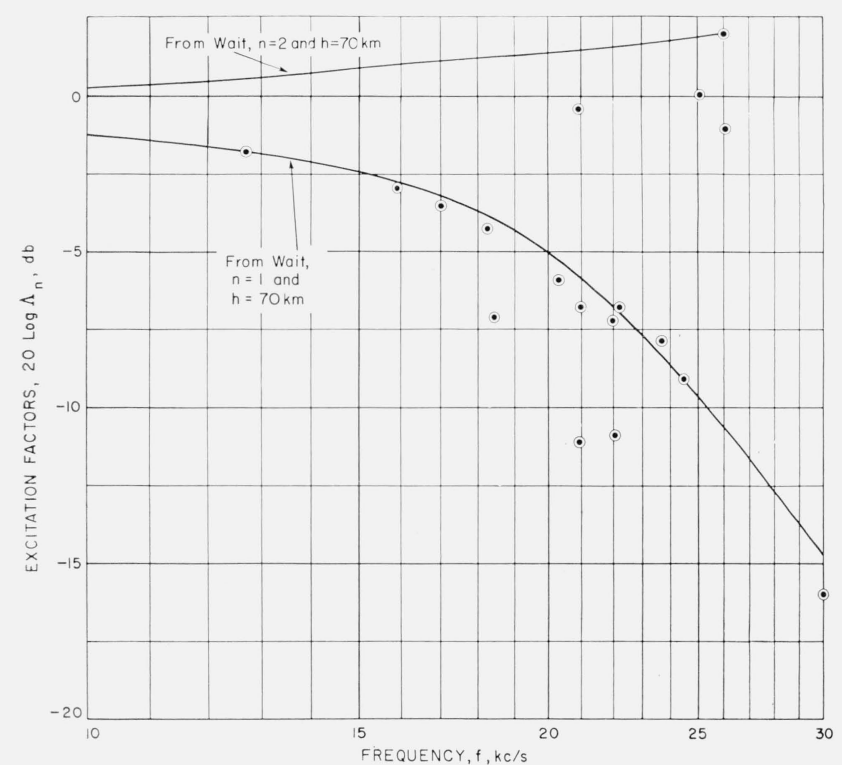

Figure 3. Excitation factors (theoretical and experimental). coupling to antennas at the earth's surface is very small. Obviously, in this case the vertical electric field versus height will not be a maximum at the earth's surface.

In the experimental data shown, an attempt was made to group the examples into propagation in both the north-south direction as compared to that in the east and the west directions. In addition, those paths which were launched over sea water and others over land were also examined. It was impossible from the rather limited data available to determine for certain any trend such as an increased excitation loss $\Lambda$ for launching over land or in any specific direction. Since, as we will see in the following section on attenuation rates that the ionospheric reflection coefficients are different for various directions of propagation, it is anticipated that a close examination of excitation factor may indicate some sensitivity in $\boldsymbol{\Lambda}$ to direction of propagation relative to the earth's magnetic field, but at present it must be concluded that we have not demonstrated this point. It is interesting to observe here that Wait and Spies [1963] in their recent descriptions of height gain for finitely conducting surfaces, show that the excitation factor shows a somewhat lower reduction, i.e., more efficient excitation, for poorly conducting earth than for perfectly conducting earth. This of course is for a condition where the whole earth's surface is uniformly poorly conducting. For the case where the guide parameters are primarily determined by a sea-ionosphere set of boundaries and the transmitter is located somewhat inland, there may be a cutback modification required which is expected to show a decrease in excitation efficiency when the antenna is situated over a finite amount of poorly conducting terrain. For normal conducting grounds at VLF this modification is expected to be rather slight; however, as frequency increases, particularly up into the LF band, this type of modification can be very pronounced as shown for aretic paths by Watt, Maxwell, and Whelan [1959].

\section{Attenuation Rates}

The effective attenuation rate used in the waveguide modal equation represents an average loss per unit distance for a discrete propagation path and time; therefore, any change in the electrical properties of the ionosphere with time and any change in the electrical properties of the earth and ionosphere over various paths will give a corresponding change in the effective attenuation rate. It is well known from work by Budden [1952], Barber and Crombie [1959], and Wait [1961] that the attenuation rate is also affected by the earth's magnetic field. This effect, which is produced by the modified electron motion in the presence of a magnetic field, may be treated as an earth's magnetic field factor $M\left(\phi_{a}\right)^{3}$ which modifies the attenuation

3 Wait uses the symbol $P$ for this factor. 
rate as follows

$$
M\left(\phi_{a}\right)=\frac{\text { Attenuation with Magnetic Field }}{\text { Attenuation without Magnetic Field }}
$$

where

$$
\begin{aligned}
& \phi_{a}=\text { azimuthal direction of propagation with } \\
& \text { respect to the horizontal component of } \\
& \text { the earth's magnetic field. }
\end{aligned}
$$

The value of $M$ is also dependent upon the geomagnetic latitude which determines the magnitude of the horizontal components of the earth's field. Since $M$ is the influence of the earth's magnetic field on VLF propagation by virtue of modification of the reflection coefficients at the ionosphere, any additional loss in the waveguide produced at the lower, i.e., earth's surface, boundary is not dependent upon $M$.

To a first approximation, the effective loss over a given propagation path $d^{\prime}$ megameters long with various effective ionosphere and ground conductivities along the path can be considered as the sum of all the incremental losses along the guide surfaces. The effective loss can be written as

$$
\alpha d / 10^{6}=\alpha d^{\prime}=\sum_{k=1}^{N_{i}} \bar{M}_{k}\left(\phi_{a}\right) \alpha_{i, k} \Delta d_{k}^{\prime}+\sum_{j=1}^{N_{g}} \Delta \alpha_{g, j} \Delta d_{j}^{\prime}
$$

where: $N_{i}$ and $N_{g}$ are the number of ionospheric and ground path increments considerəd, $M_{k}$ $\left(\phi_{a}\right) \alpha_{i, k} \Delta d_{k}^{\prime}$ is the loss contributed by the ionosphere over the incremental distance $\Delta d_{k}^{\prime}$, and $\Delta \alpha_{g, j} \Delta d_{j}^{\prime}$ is the loss contributed by the earth over the incremental distance $\Delta d_{j}{ }^{\prime}$. For paths where the boundary surfaces can be considered as uniform, the attenuation rate becomes

$$
\alpha=M\left(\phi_{a}\right) \alpha_{i}+\Delta \alpha_{g}
$$

The contribution due to ground loss can be shown to be related to ground conductivity by the expression obtainable from Wait [1957]

$$
\Delta \alpha_{g}[\mathrm{db} / \mathrm{Mm}] \simeq 46 f^{1 / 2} \sigma^{-1 / 2} h^{-1}:
$$

where: $f$ is the frequency in cycles/second, $\sigma$ is the ground conductivity in mhos/meter, and $h$ is the effective ionospheric height in meters. This expression is valid only when $f$ is low enough that the mode is not becoming earth detached; i.e., about $15 \mathrm{kc} / \mathrm{s}$ or less for $n=1$. The conductivity value must also not be too low for this relation to apply. The earth detached mode may be seen by the more rigorous computations of $\Delta \alpha_{g}$ by Spies and Wait [1961] in which a maximum value of $\Delta \alpha_{g}$ is reached with an increase of frequency, and a further increase will decrease $\Delta \alpha_{g}$.

A great deal of work has been conducted in calculating the attenuation rates of the ionosphere and earth of different conductivities by a number of authors. The results are presented either in the form of attenuation rate or in the form of reflection coefficients at the earth and ionosphere.

The attenuation rate can be obtained from the reflection coefficients as a perturbation solution which assumes that

$$
\alpha[\mathrm{db} / \mathrm{Mm}] \simeq \frac{R_{g}[\mathrm{db}]+R_{i}[\mathrm{db}]}{2 d_{1}^{\prime}}
$$

where: $R_{g}$ and $R_{i}$ are the reflection coefficients of the ground and ionosphere respectively, and $2 d_{1}^{\prime}$ is the average distance in megameters containing one ground and one ionosphere reflection at mode resonance. An approximate solution for $d_{1}$ that is valid over an appreciable range of frequencies yields

$$
\begin{aligned}
& \alpha[\mathrm{db} / \mathrm{Mm}] \simeq 1.2 \times 10^{13}\left(R_{g}[\mathrm{db}]\right. \\
& \left.\quad+R_{i}[\mathrm{db}]\right)\left(2 \pi n-\phi_{g}-\phi_{i}\right) / f h^{2}
\end{aligned}
$$

where $\phi_{g}$ and $\phi_{i}$ are the phase lag upon reflection at the ground and ionosphere, respectively.

Values of $R_{i}$ from Wait and Walters [1963] are shown in figure 4 as a function of frequency and parametric in the term $l$ which is the conductivity gradient of the ionosphere. ${ }^{4}$ Typical conductivity gradients are shown in figure 5 for both day and night. The conductivity gradient term, $l$, may be calculated from these curves by the following relationship:

$$
l=\frac{z-z_{0}}{2.3 \log \left(\sigma / \sigma_{0}\right)}
$$

where $z_{0}$ and $z$ are the ionosphere heights corresponding to the conductivities $\sigma_{0}$ and $\sigma$, respectively.

The ionospheric conductivity neglecting the earth's magnetic field is given by Jordan [1950] as

$$
\begin{aligned}
\sigma & =\frac{N e^{2} \nu}{m\left(\nu^{2}+\omega^{2}\right)} \\
& \simeq 2.78 \times 10^{-8} N / \nu
\end{aligned}
$$

where: $e$ and $m$ are the electron charge and mass, $N$ is the number of electrons per cubic meter, and $\nu$ is the effective collisional frequency in collisions per second. Typical daytime and nighttime values of the conductivity gradient are $l_{\mathrm{day}} \simeq 2.6 \mathrm{~km}$ and $l_{\text {night }} \sim 1.5 \mathrm{~km}$.

Anticipated sea water path attenuation rates can be calculated with the aid of (3.6) assuming that $f=20 \mathrm{kc} / \mathrm{s}, \quad R_{g} \simeq 0 \mathrm{db}, \quad \phi_{g}=0, \quad h_{\text {day }}=70 \mathrm{~km}$, and $h_{\text {night }}=90 \mathrm{~km}$. From figure $4, R_{i \text { day }} \simeq 4.5 \mathrm{db}$ and $R_{i \text { night }}=3.5 \mathrm{db}$, and from Wait and Walters [1963], $\phi_{i \mathrm{day}}=2.4$ and $\phi_{i \mathrm{night}}=2.7$. The resulting calculated attenuation rates are

$$
\begin{aligned}
& \alpha_{\text {day }}=2.2 \mathrm{db} / \mathrm{Mm} \\
& \alpha_{\text {night }}=0.9 \mathrm{db} / \mathrm{Mm} .
\end{aligned}
$$

${ }^{4}$ Note that Wait uses the parameter $\beta$ which equals $1 / l$. One of the most significant recent contributions to VLF propagation theory is contained in the paper, "Reflection of electromagnetic waves from a lossy magneto plasma," by J. R. Wait and L. C. Walters [1963a]. The model employed in this paper is a close representation of what we presently expect for the ionosphere. The resultant values of $R_{i}$ now reffect the influence of the earth's magnetic field as well as finite conductivity gradients. 


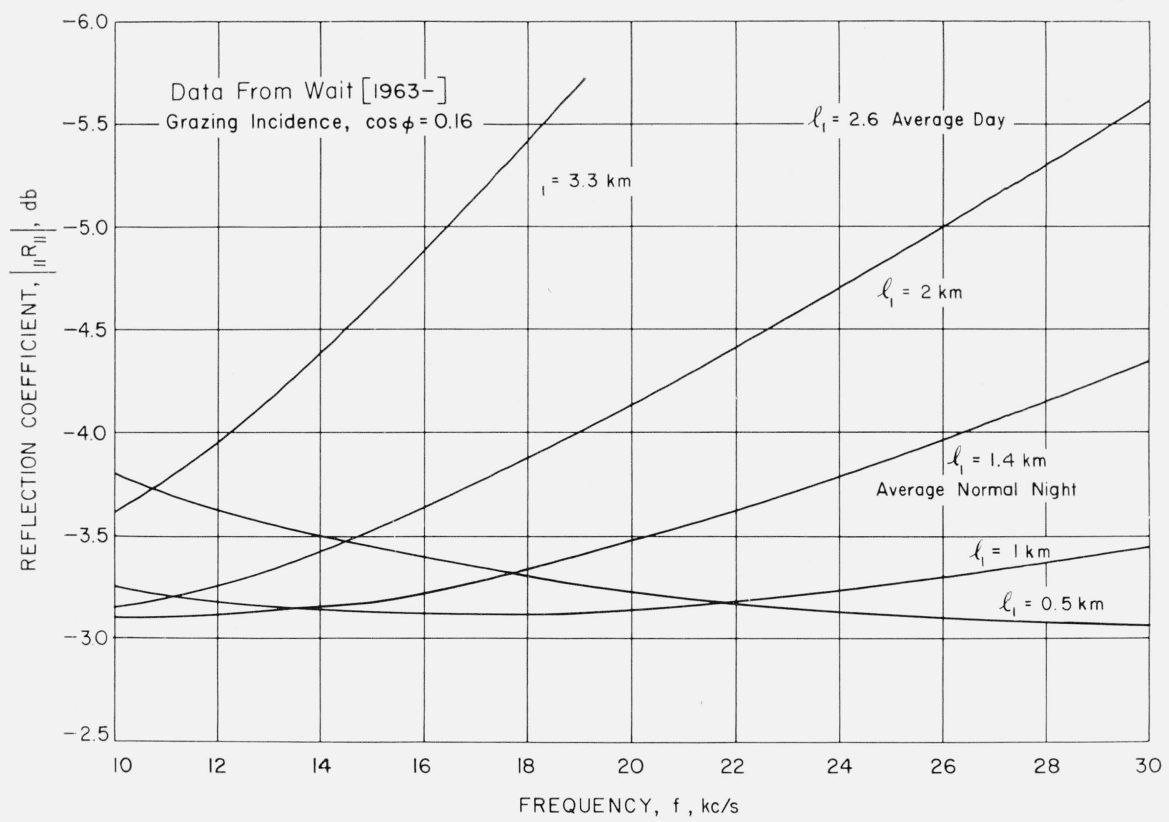

FiguRE 4. Reflection coefficients versus frequency for nonsharply bounded ionosphere.

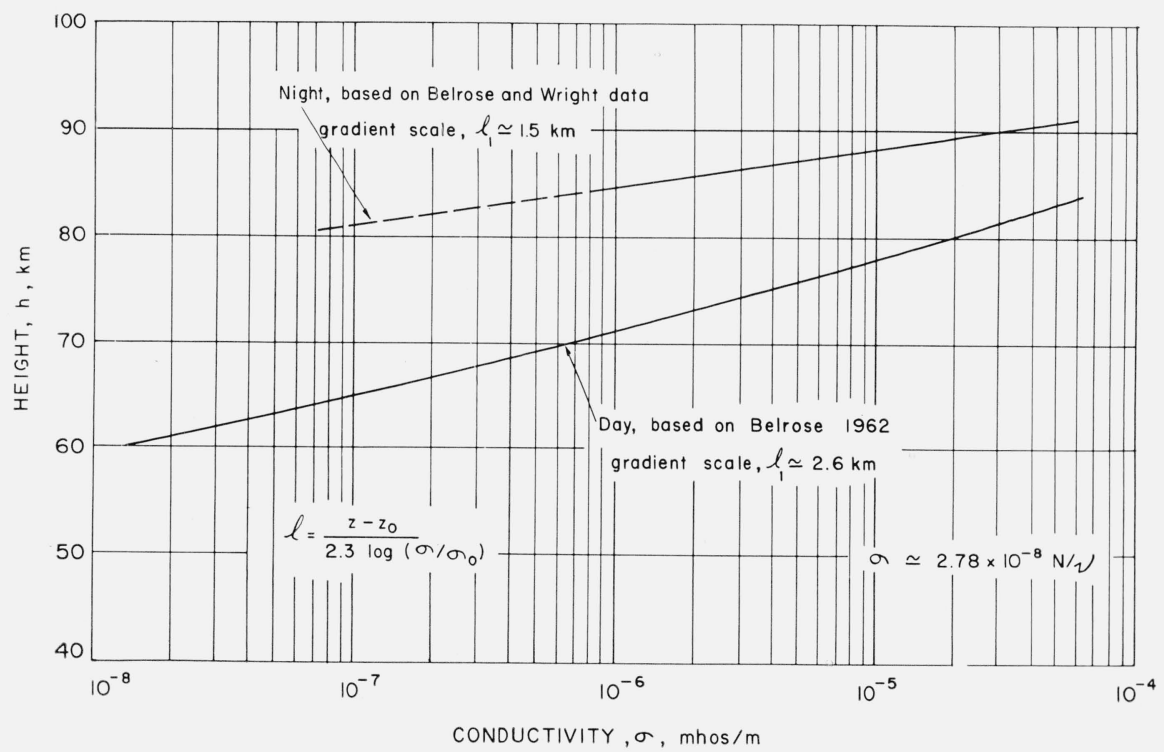

FIGURE 5. Typical ionosphere conductivity gradients.

Curves of experimentally determined daytime values of attenuation rates are shown in figure 6 as a function of frequency and parametric in direction of propagation. Much of the data for this figure came from curves similar to figure 2 . The curves were drawn through the average of all the points within a plus or minus $45^{\circ}$ sector of the given direction.

For frequencies above $25 \mathrm{kc} / \mathrm{s}$, existing data is contaminated by higher order mode contributions. Because of this, the dashed portions of the curves are based primarily on theoretical values by Wait and Walters [1963a].

Wait [1964] has calculated the change in attenuation rate produced by an exponential ionosphere relative to that previously calculated using a sharply bounded ionosphere [Spies and Wait, 1961]. The resulting exact solutions of $\alpha$ for an exponential ionosphere ( $h=70 \mathrm{~km}$ day and $90 \mathrm{~km}$ night) may be seen in figures 7 and 8 for the first and second order mode respectively. The curves were obtained by using typical values of the conductivity gradient 


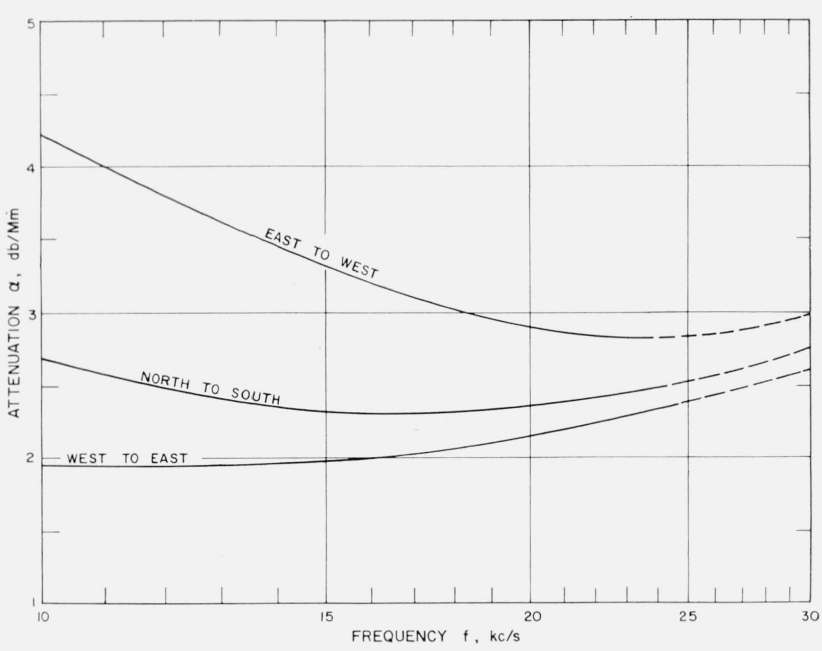

FigURE 6. Attenuation rates for predominately sea water paths versus frequency for moderate and low sunspot numbers (experimental).

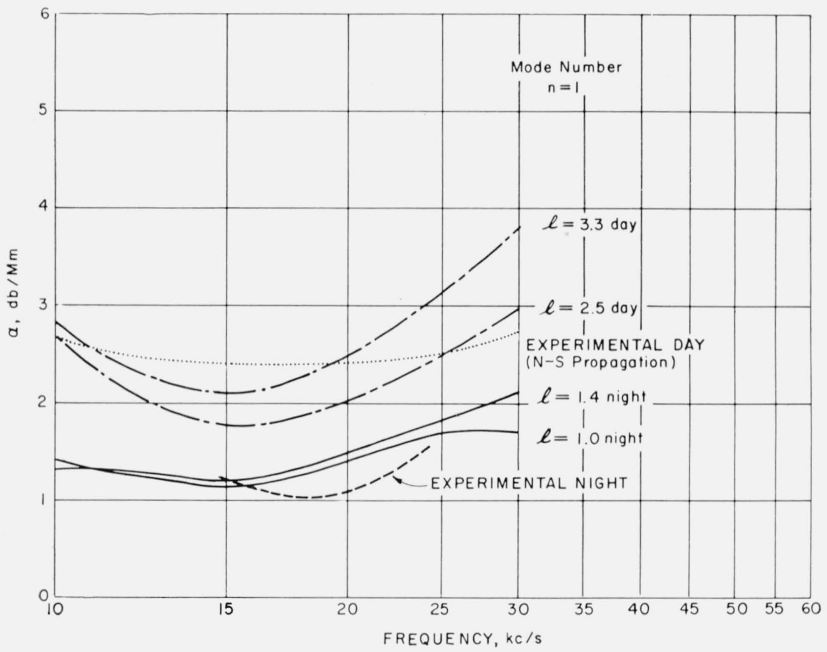

FIGU RE 7. Attenuation rates over predominately sea water paths for $\mathrm{n}=1$ (experimental and theoretical).

expected for day and night. Indicated on the curves are experimentally determined values of attenuation rate. It can be seen that the theoretical values are in good agreement with those obtained by experimentally collected data for the first order mode. For the second order mode, the experimental values obtained are much more uncertain which may account for the only fair agreement during the night. This uncertainty may be due to the randomness of nighttime propagation conditions; i.e., variable $l$ and $h$. In addition, the distance involved in which the second order mode is predominate is usually very short for accurate $\alpha$ determinations.

\section{Field Strength Versus Distance}

Figure 9 shows the observed vertical electrical field strength as a function of frequency for a typical

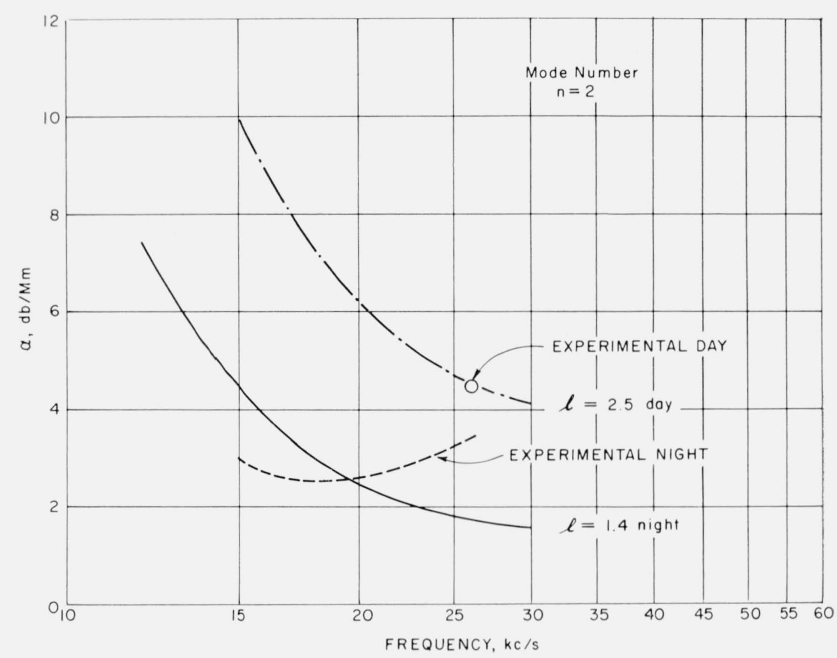

Figure 8. Attenuation rates over predominately sea water paths for $\mathrm{n}=2$ (experimental and theoretical).

west to east propagation path. Also shown on the figure are the calculated groundwave and modal fields using values of the excitation factor $\Lambda$ as shown in figure 3 and expected values of attenuation as shown for $n=1$ in figure 6 and $n=2$ in figure 8 . At distances less than $300 \mathrm{~km}$, the groundwave is predominate and is practically independent of frequency over the VLF range. This may be seen from the matching of data points at 20 and $16 \mathrm{kc} / \mathrm{s}$. At approximately $100 \mathrm{~km}$, an interference pattern begins to appear which is likely due to interference between the groundwave and the first hop skywave. Near $600 \mathrm{~km}$, the groundwave and skywave are approximately of equal amplitude. Since their phase versus distance rates are different, a strong cancellation, or null, is expected in this region.

Beyond $1 \mathrm{Mm}$ where we are now considering mode theory, the first order mode is apparently predominate and has an attenuation rate of approximately $2 \mathrm{db} / \mathrm{Mm}$. Beyond about $16 \mathrm{Mm}$, it can be seen that the converging energy due to antipode focusing actually increases the fields at a greater rate than the loss due to attenuation. This buildup in field strength continues until the antipode is reached. It can be seen that the observed antipode value [Garner, 1962] is very close to the calculated value. Included on the figure are the unattenuated mode fields for the first and the second order modes.

If the transmitted frequency is increased, the attenuation of the second order mode will decrease rapidly and at the same time the excitation of the first order mode will decrease. It then is apparent that as the frequency increases the second order mode will predominate to greater ranges. Figure 10 is a curve of vertical field strength as a function of frequency for east to west propagation for data in the 21 to $25 \mathrm{kc} / \mathrm{s}$ region. The excitation factors were obtained from figure 3 and the attenuation rates were chosen to best fit the data. The first large null in 


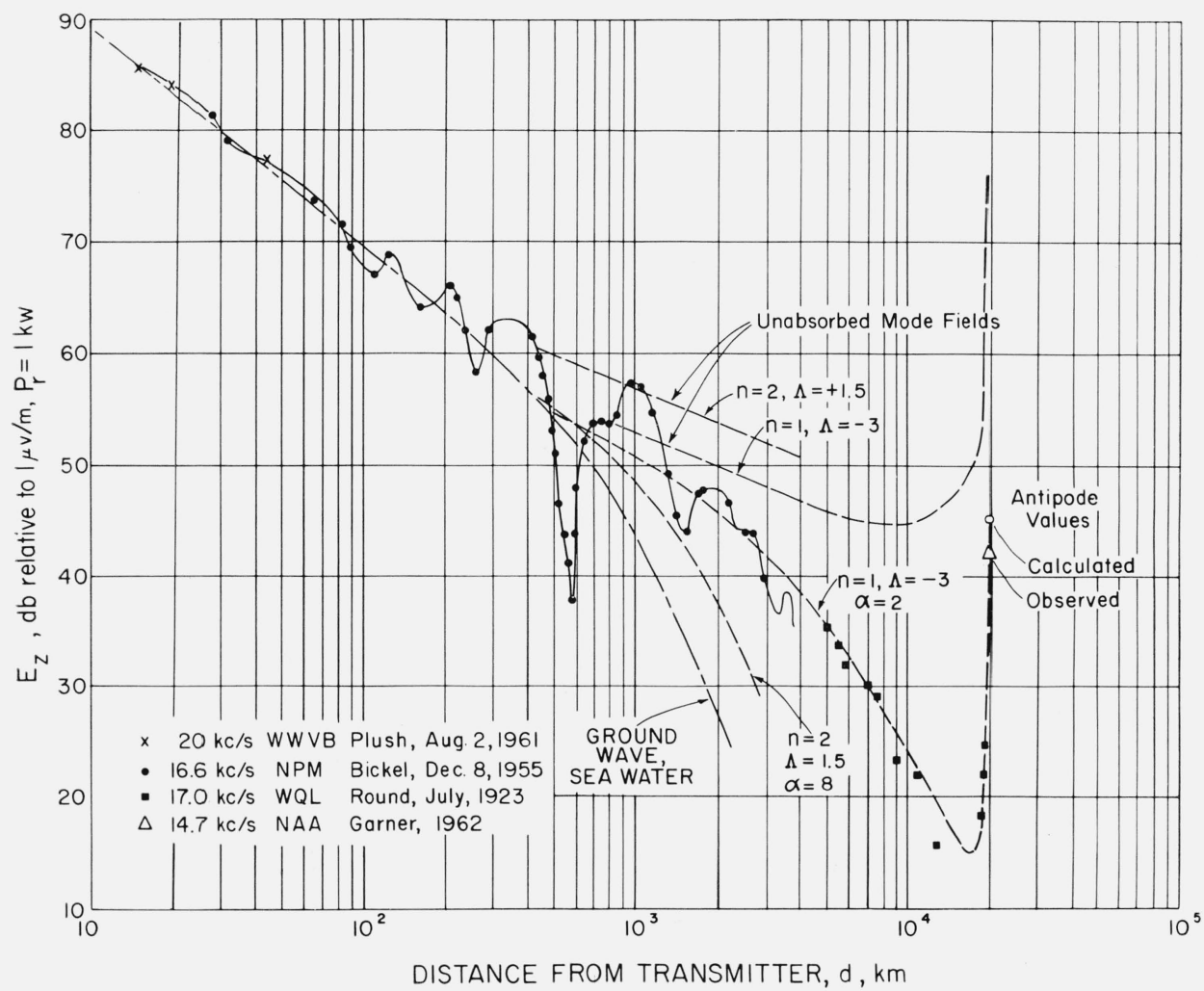

Figure 9. Field strength versus distance at $16 \mathrm{kc} / \mathrm{s}$ for propagation to the east.

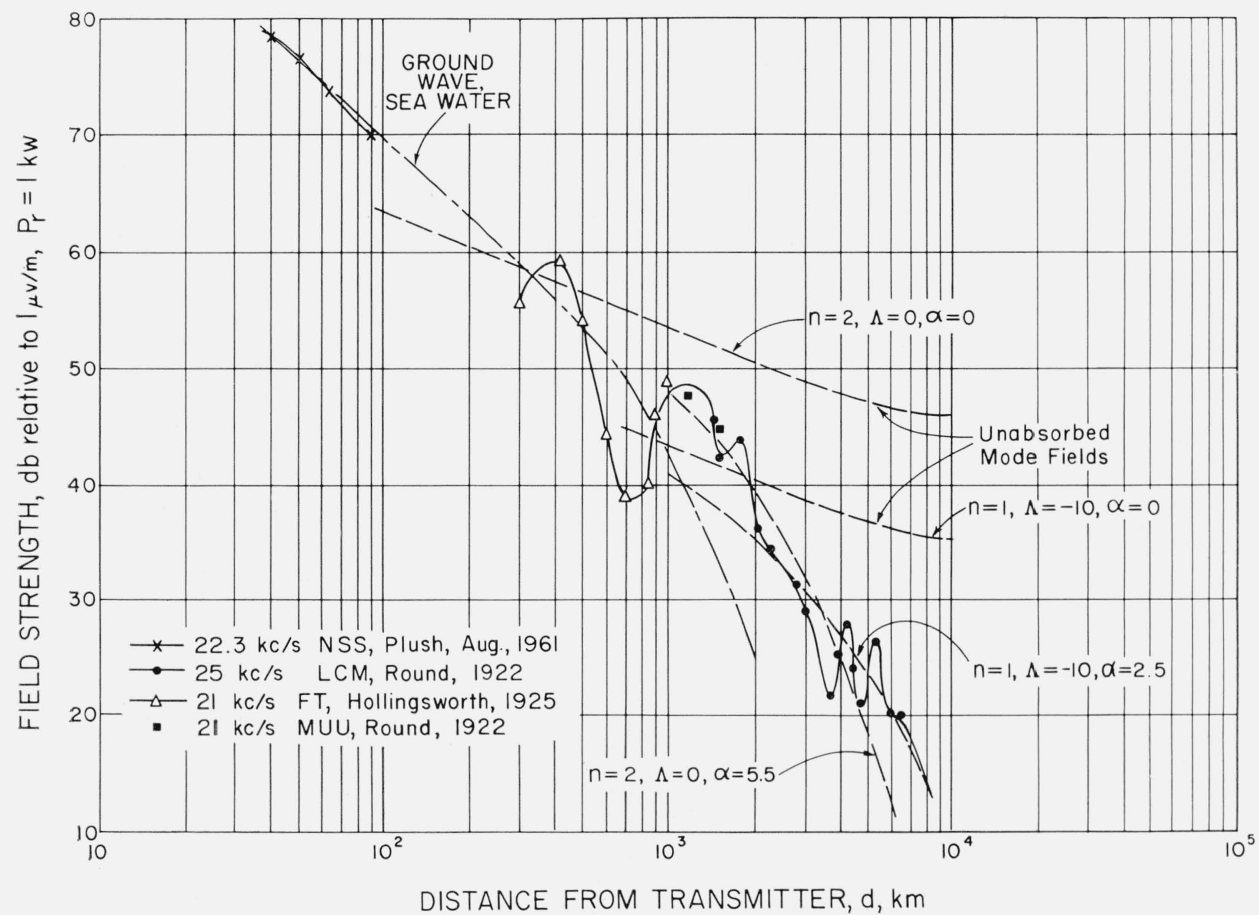

Figure 10. Field strength versus distance at $25 \mathrm{kc} / \mathrm{s}$ for propagation to the west. 
field strength is now seen to occur at $700 \mathrm{~km}$ and is again likely caused by cancellation between the groundwave and the first hop skywave.

Considering mode theory for distances greater than $1 \mathrm{Mm}$, the second order mode appears to be the predominate mode out to about $3 \mathrm{Mm}$ where interference now occurs between the first and second order modes. Beyond $4 \mathrm{Mm}$ the first order mode appears to be predominate. On some occasions, the second order mode has been observed to be predominate at nighttime for distances up to $6 \mathrm{Mm}$ or more for frequencies above $18 \mathrm{kc} / \mathrm{s}$.

The authors gratefully acknowledge information obtained during helpful discussions with J. R. Wait, R. W. Plush, W. E. Gustafson, J. A. Pierce, M. L. Tibbals, W. E. Garner, and F. J. Rhoads. The assistance of Mrs. W. Mau in preparation of the manuscript and C. W. Vogt and E. F. Oberteuffer in preparing the illustrations is also acknowledged.

\section{References}

Barber, N. F., and D. D. Crombie (1959), VLF Reflections from the ionosphere in the presence of a transverse magnetic field, J. Atmos. Terrest. Phys. 16, 37.

Budden, K. G. (1952), The propagation of a radio-atmospheric, Phil. Mag. 43, 1179-1952.

Garner, W. E., F. J. Rhoads, J. E. Bickel, and R. W. Plush (1962), Measured amplitude and phase of the antipodal fields of a VLF transmitter, paper presented at Fall URSI Meeting, Ottawa, Canada, 15-17 Oct.

Jordan, E. C. (1950), Electromagnetic Waves in Radiating Systems (Prentice-Hall, Englewood Cliffs, N.J.).
Round, H. J., T. L. Eckersley, K. Tremellen, and F. C. Lunnon (Oct. 1925), Report on measurements made on signal strength at great distances during 1922 and 1923 by an expedition sent to Australia, IEE J. 63, No. 346.

Spies, K. P., and James R. Wait (July 17, 1961), Mode calculations for VLF propagation in the earth-ionosphere

wave guide, NBS Tech. Note No. 114.

Wait, J. R. (June 1957), The mode theory of VLF ionospheric propagation for finite ground conductivity, Proc. IRE 45, No. 6, 760-767.

Wait, J. R. (1958/III), A study of VLF field strength databoth old and new, Geofisica Pura e Applicata-Milano, 41, 73-85.

Wait, J. R. (Mar.-Apr. 1960), Terrestrial propagation of very-low-frequency radio waves, a theoretical investigation, J. Res. NBS 64D (Radio Prop.), No. 2, 153-204.

Wait, J. R. (Jan.-Feb. 1961), A new approach to the mode theory of VLF propagation, J. Res. NBS 65D (Radio Prop.), No. 1, 37-46.

Wait, J. R. (1962), Electromagnetic Waves in a Stratified Media (Pergamon Press, Oxford).

Wait, J. R. (July-Aug. 1963), Influence of the lower ionosphere on propagation of VLF waves at great distances, J. Res. NBS 67D (Radio Prop.), No. 4, 375-381.

Wait, J. R., and L. C. Walters (May-June 1963), Reflection of VLF radio waves from an inhomogeneous ionosphere. Part I. Exponentially varying isotropic model, J. Res.

NBS 6yD (Radio Prop.), No. 3, 361-367.

Wait, J. R., and L. C. Walters (Jan.-Feb. 1964), Reflection of electromagnetic waves from a lossy magnetoplasma, J. Res. NBS 68D (Radio Prop.), No. 1, 97.

Wait, J. R., and K. P. Spies (Mar.-Apr. 1963), Height gain for VLF radio waves, J. Res. NBS 6\%D (Radio Prop.), No. 2, 183-187.

Watt, A. D., E. L. Maxwell, and E. H. Whelan (July-Aug. 1959), Low frequency propagation paths in aretic areas. J. Res. NBS 63D (Radio Prop.), No. 1, 99-112.

(Paper 68D1-307) 